\title{
Prolidase deficiency: a new genetic cause of combined pulmonary fibrosis and emphysema syndrome in the adult
}

\author{
To the Editor:
}

Pulmonary fibrosis is considered to result from recurrent alveolar epithelial injury coupled with dysfunctional alveolar wound healing mechanisms, some of which have a genetic background. Pulmonary fibrosis in the adult has not been previously associated with prolidase deficiency, an innate deficiency of amino acid metabolism.

A 22-year old Portuguese man was referred because of digital clubbing present since the age of 12 years. He had no familial history. He had smoked 20 cigarettes per day since the age of 13 . He was short of breath at exercise, with no cough. Velcro crackles were present at lung auscultation. The chest computed tomography (CT) demonstrated infiltrative lung disease, with mid- and upper lobe predominance, consisting of reticulation, and septal and peribronchovascular thickening (figure 1). Mediastinal and axillar lymphadenopathies were present. Liver and spleen were increased in diameter. Bronchoalveolar lavage demonstrated 10\% lymphocytes, $8 \%$ neutrophils, and $82 \%$ macrophages. No foamy macrophages were reported. Forced vital capacity (FVC) was $93 \%$ of predicted value, forced expiratory volume in $1 \mathrm{~s}\left(\mathrm{FEV}_{1}\right)$ $94 \%$ of predicted, $\mathrm{FEV}_{1} / \mathrm{FVC} 0.83$, total lung capacity (TLC) $95 \%$, diffusion capacity for carbon monoxide ( $\left.D_{\mathrm{LCO}}\right) 83 \%$ and transfer coefficient $\left(K_{\mathrm{CO}}\right) 78 \%$. Blood gas was normal. Routine blood tests were normal, except for increased lacticodehydrogenase and gammaglobulins $\left(81 \mathrm{~g} \cdot \mathrm{L}^{-1}\right)$. Serology for HIV was negative.

1 year later, lung function had declined. A right-sided videothoracoscopic lung biopsy was performed in three lobes, demonstrating diffuse alveolar fibrosis with collagen deposition, architectural distortion and alveolar cysts, which did not fulfil criteria for usual interstitial pneumonia. Dense lymphocytic inflammation was present with bronchiolar and peribronchovascular predominance, together with lymphoid follicles and germinative centres. Bronchiolar metaplasia was present. Fibroblastic foci were not found. Cholesterol clefts were abundant. In addition, airspaces were filled by patchy, granular, eosinophilic proteinaceous exudate, stained with periodic acid Schiff, suggesting secondary pulmonary alveolar proteinosis accompanying pulmonary fibrosis. Immunohistochemistry demonstrated strong staining of alveolar exudate with anti-surfactant protein- $\mathrm{C}$ antibody. Birefringent particles were not found under polarised light. Staining of elastic fibres was normal. The pleura was normal except for mild lymphocytic inflammation; a mediastinal lymph node was normal. Anti-granulocyte-macrophage colony-stimulating factor antibodies were negative. The patient was advised to quit smoking and corticosteroids were initiated, together with treatment for osteoporosis.

Examination further demonstrated small body size, normal body weight, kyphosis, dysmorphic face, grey hair, flat feet, some cognitive retardation, and numerous scars from dermal cysts. The patient was diagnosed with leg ulcer dermatitis, axonal neuropathy, and non-erosive, bilateral symmetric synovitis, osteoporosis, mixed glomerular and tubular proteinuria $\left(0.5 \mathrm{~g} \cdot \mathrm{L}^{-1}\right)$, and mild-severity autoimmune hepatitis. Rheumatoid factor, homogeneous antinuclear antibodies, and mixed type-II cryoglobulinaemia were present. Overlapping rheumatoid arthritis and systemic lupus were diagnosed.

Sequencing of TERT, TERC, SFTPC, SFTPB and ABCA3 genes did not show pathogenic variants. Analysis of blood and urine amino acids demonstrated massive iminodipeptiduria. The level of glycine-proline dipeptide was $30 \mu \mathrm{mol} \cdot \mathrm{L}^{-1}$ in the plasma (normal: absent) and $8480 \mu \mathrm{mol} \cdot \mathrm{L}^{-1}$ in urine (normal: $<20 \mu \mathrm{mol} \cdot \mathrm{L}^{-1}$ ). The biochemical diagnosis of prolidase deficiency was established. PEPD gene sequencing identified the homozygous variant NM_000285.4:c.692_694del (p.Tyr231del) in exon 10, previously reported in two

@ERSpublications

Prolidase deficiency is a new genetic cause of combined pulmonary fibrosis and emphysema syndrome in the adult http://bit.ly/2QRgqMH

Cite this article as: Cottin V, Nasser M, Traclet J, et al. Prolidase deficiency: a new genetic cause of combined pulmonary fibrosis and emphysema syndrome in the adult. Eur Respir J 2020; 55: 1901952 [https://doi.org/10.1183/13993003.01952-2019]. 

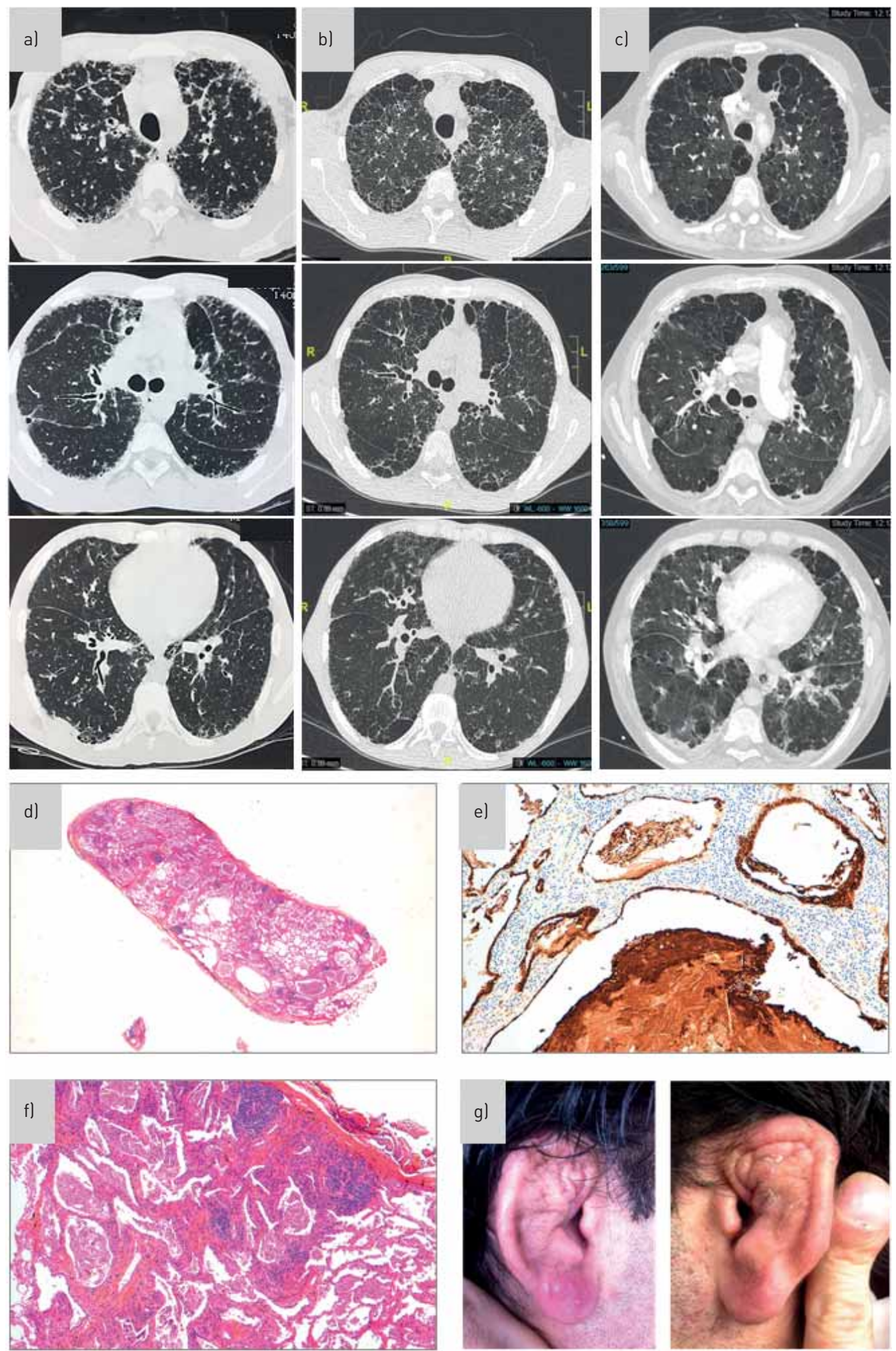

FIGURE $1 \mathrm{a}-\mathrm{c}$ ) Lung window of axial chest computed tomography demonstrating infiltrative lung disease, with mid and upper lobe predominance, with reticulation, and septal and peribronchovascular thickening, at baseline (a), year +9 (b) and year +17 (c). c) At last follow-up, fibrotic features are less pronounced, whereas cystic changes, emphysematous lesions and air trapping have worsened. d-f) Microscopic examination of right-sided lung biopsy. d) Homogeneous distribution of alveolar wall thickening and of eosinophilic proteinaceous exudate (hemalun eosinin saffron (HES) $\times 1.5$ ). e) Intense cytoplasmic staining of epithelial alveolar cells and of alveolar exudate with anti-surfactant protein $C$ antibody (immunohistochemistry $\times 40$ ). f) Alveolar wall thickening and eosinophilic proteinaceous exudate; two lymphoid follicles are also visible (HES $\times 10) . g$ ) Deformation of ear lobes on both sides resulting from deformity and chronic inflammation due to relapsing polychondritis. 
unrelated Portuguese patients [1], but never observed in GnomAD populations. This in-frame 3bp-deletion impacts the secondary structure of the protein and strongly reduces prolidase enzymatic activity [2]. Oral supplementation with vitamin $\mathrm{C}$, proline and essential amino acids was initiated.

On follow-up, he continued smoking 40 cigarettes per day, and 2 joints of cannabis per day for 11 years. The patient was admitted on multiple times for lower airway infections. Oral corticosteroid treatment was maintained despite limited patient compliance, with some transient benefit observed on clinical and functional outcome. 6 years after presentation, he developed atrophic polychondritis with typical relapsing inflammation and deformity of ear cartilage. Over the years, lung function deteriorated, with severe airflow obstruction and air trapping. 10 years after the lung biopsy, FVC was $63 \%$ of predicted, $\mathrm{FEV}_{1} 38 \%, \mathrm{FEV}_{1} /$ FVC 0.39, residual volume 5.6 L (233\%), residual volume (RV)/TLC 0.67 (248\%), TLC 139\%, $D_{\text {LCO }} 48 \%$, and $K_{\mathrm{CO}} 48 \%$. Hypercarbia was then present, and supplemental oxygen therapy and noninvasive ventilation were initiated, with excellent tolerance. At last follow-up 16 years after lung biopsy, FVC was 49\%, FEV 1 $19 \%, \mathrm{FEV}_{1} / \mathrm{FVC} 0.32$, TLC $121 \%$, RV 295\%, RV/TLC 0.72, $D_{\mathrm{LCO}} 14 \%, K_{\mathrm{CO}} 23 \%$. Chest CT demonstrated progression of interstitial changes over the first 10 years of follow-up, with later apparent decreased severity of interstitial abnormalities at imaging while emphysematous changes markedly progressed.

Prolidase deficiency (OMIM \#170100) is a rare autosomal recessive multisystem disorder associated with imidodipeptiduria and reduced prolidase activity, caused by homozygous or compound heterozygous mutation in the PEPD gene on chromosome 19q13. The disorder is clinically heterogeneous and may comprise slow healing ulcerations of the legs and feet, various types of eruptions, telangiectases, mental retardation, recurrent respiratory tract infections, and facial dysmorphism [3]. Diagnosis is based on biochemical techniques [4].

Previous reports in prolidase deficiency either were related to children [5] or did not provide detail about the phenotype of lung disease, which may include recurrent pulmonary infections, cystic changes, bronchiectases, diffuse ground glass attenuation, minor linear atelectasis [6] and autoimmune pulmonary capillaritis [5]. Our adult patient had a non-usual interstitial pneumonia pattern of pulmonary fibrosis at biopsy. Cholesterol clefts were prominent similar to paediatric cases [5]. In addition to collagen deposition and alveolar cyst formation, significant lymphocytic bronchiolitis was observed, which may have contributed to air trapping, development of low attenuation areas, and severe airflow obstruction, which became prominent over time despite long-term oral corticosteroids. Indeed, long-term follow-up suggests that fibrosis may surprisingly have become less pronounced with time, while emphysema and cystic lesions progressed and likely were responsible for chronic respiratory insufficiency, with prolonged efficacy of noninvasive ventilation. Although the imaging features were that of combined pulmonary fibrosis and emphysema, pulmonary function demonstrated progressive airflow obstruction with increased residual volume not characteristic of this syndrome. Interestingly, other genetic causes of combined pulmonary fibrosis and emphysema have been described [7]. Corticosteroids initiated because of presence of inflammatory infiltrates on the biopsy provided some medium-term improvement but were discontinued after 8 years because of severe osteoporosis, and may have contributed to recurrent respiratory infections.

Pathogenesis of fibrosis and emphysema in prolidase deficiency is unclear. Tobacco smoking may have played a role. Cell death of fibroblasts [8] with interference with the normal recycling of collagen [9], and increased oxidative stress [10] may contribute to both fibrosis and emphysema. This observation suggests that prolidase deficiency is a genetic factor that may contribute to pulmonary fibrosis and potentially emphysema.

Vincent Cottin $\oplus^{1}$, Mouhamad Nasser $\oplus^{1}$, Julie Traclet ${ }^{1}$, Lara Chalabreysse ${ }^{2}$, Anne-Sophie Lèbre $\oplus^{3}$, Salim Si-Mohamed ${ }^{4}$, François Philit ${ }^{5}$ and Françoise Thivolet-Béjui ${ }^{2}$

${ }^{1}$ National Coordinating Reference Centre for Rare Pulmonary Diseases, Louis Pradel Hospital, Hospices Civils de Lyon, UMR 754, Claude Bernard University Lyon 1, member of OrphaLung, RespiFil, and ERN-LUNG, Lyon, France. ${ }^{2}$ Dept of Pathology, Groupement Hospitalier Est, Hospices Civils de Lyon, Claude Bernard University Lyon 1, Lyon, France. ${ }^{3}$ CHU Reims, Hôpital Maison Blanche, Pôle de Biologie, Service de Génétique, Reims, France. ${ }^{4}$ Dept of Radiology, Louis Pradel Hospital, Hospices Civils de Lyon, Lyon, France. ${ }^{5}$ Dept of Respiratory Medicine, Croix Rousse Hospital, Hospices Civils de Lyon, Lyon, France.

Correspondence: Vincent Cottin, Service de Pneumologie, Hôpital Louis Pradel, 28 avenue Doyen Lepine, F-69677 Lyon Cedex, France. E-mail: vincent.cottin@chu-lyon.fr

Received: 10 Oct 2019 | Accepted after revision: 19 Dec 2019

Acknowledgements: We thank Sylvie Boyer (Lyon) for aminoacids measurement, and Kais Ahmad, Jade Cuilleron, Sylvie Ernesto, Laure Folliet, Nathalie Guffon, Lize Kiakouama, Jacques-Olivier Maillard, Pascale Nesme-Meyer and Judith Proovost and for clinical care of the patient.

Ethics statement: This study complied with the Declaration of Helsinki. Written informed consent was obtained for this report. French law does not require ethics committee approval or informed consent for retrospective data collection. The patient has provided written informed consent for the publication of the present manuscript as per guidelines of the journal. 
Author contributions: All authors fulfil the ICMJE criteria for authorship. V. Cottin was responsible for design, analysis and interpretation of data. All authors participated to data collection. V. Cottin prepared the manuscript. All authors were responsible for manuscript review and revision. All authors approved the final version of the manuscript. The corresponding author (V. Cottin) had full access to all data in the study and had final responsibility for the decision to submit for publication.

Conflict of interest: None declared.

\section{References}

1 Lupi A, De Riso A, Della Torre S, et al. Characterization of a new PEPD allele causing prolidase deficiency in two unrelated patients: natural-occurrent mutations as a tool to investigate structure-function relationship. J Hum Genet 2004; 49: 500-506.

2 Besio R, Gioia R, Cossu F, et al. Kinetic and structural evidences on human prolidase pathological mutants suggest strategies for enzyme functional rescue. PLoS One 2013; 8: e58792.

3 Lupi A, Tenni R, Rossi A, et al. Human prolidase and prolidase deficiency: an overview on the characterization of the enzyme involved in proline recycling and on the effects of its mutations. Amino Acids 2008; 35: 739-752.

4 Kurien BT, Patel NC, Porter AC, et al. Prolidase deficiency and the biochemical assays used in its diagnosis. Anal Biochem 2006; 349: 165-175.

5 Rayment JH, Jobling R, Bowdin S, et al. Prolidase deficiency diagnosed by whole exome sequencing in a child with pulmonary capillaritis. ERJ Open Res 2019; 5: 00205-2018.

6 Nir V, Ilivitky A, Hakim F, et al. Pulmonary manifestations of prolidase deficiency. Pediatr Pulmonol 2016; 51: 1229-1233.

7 Cottin V, Cordier JF. The syndrome of combined pulmonary fibrosis and emphysema. In: Cottin V, Cordier JF, Richeldi L, eds. Orphan lung disease: A clinical guide to rare lung disease. London, Springer Verlag, 2015; pp. 327-347.

8 Forlino A, Lupi A, Vaghi P, et al. Mutation analysis of five new patients affected by prolidase deficiency: the lack of enzyme activity causes necrosis-like cell death in cultured fibroblasts. Hum Genet 2002; 111: 314-322.

9 Jackson SH, Dennis AW, Greenberg M. Iminodipeptiduria: a genetic defect in recycling collagen; a method for determining prolidase in erythrocytes. Can Med Assoc J 1975; 113: 762-763.

10 Verma AK, Raj J, Sharma V, et al. Plasma prolidase activity and oxidative stress in patients with Parkinson's disease. Parkinsons Dis 2015; 2015: 598028. 